\title{
Zur Kenntnis biologisch wichtiger Oxydationen.
}

III. Mitteilung.

Von

Hans Euler und Ivan Bolin.

(Aus dem biochemischen Laboratorium der Hochschule Stockholm.)

(Der Redaktion zugegangen am 23. Juni 1909.)

Preßt man die frischen Blätter von Luzernen (Medicago sativa), welche während der Blütezeit geerntet worden sind, stark aus, und fällt den Saft mit Alkohol, so gewinnt man eine Substanz, welche zusammen mit geringen Mengen neutraler Mangansalze die Oxydation von Polyphenolen durch Sauerstoff katalytisch beschleunigt. Der dabei wirksame Bestandteil ist nach Bertrand eine Laccase, d.h. er wäre der im Milchsaft von Rhus vernicifera enthaltenen Oxydase an die Seite zu stellen.

$\mathrm{Da}$ die Vermutung geäußert worden ist (Ruff, Oppenheimer, ${ }^{1}$ ) Trillat $\left.{ }^{2}\right)$ u. a.), daß der katalytische Effekt der Oxydasen im wesentlichen auf deren Mangangehalt zurückzuführen sei - eine Vermutung, welche sehr nahe liegt, da ja Mangan in alkalischer Lösung als kräftiger Oxydationskatalysator lange bekannt ist - so haben wir zunächst die Oxydationsgeschwindigkeit im System Mangansalz - Alkali - Hydrochinon genauer untersucht, ${ }^{3}$ ) um zu ermitteln, ob dessen Wirkungen mit denjenigen von Oxydase quantitativ vergleichbar seien. Es wurde zunächst festgestellt, daß die Wirkung der Medicago-Laccase nicht auf die Wirkung $\mathrm{Mn}_{\ddagger}^{+}$und $\mathrm{OH}^{-}$zurückgeführt werden kann, und anderseits gezeigt, daß die Alkali- und Erdalkalisalze aliphatischer Oxysäuren den gleichen Effekt wie die sogenannte Medicago-Laccase hervorbringen.

1) Die Fermente, 2. Aufl., S. 367.

$\left.{ }^{2}\right)$ Compt. rend., Bd. CXXXVIII, S. 94 und 274 (1904).

3) Diese Zeitschrift, Bd. LVII, S. 80 [1908]. 
In einer zweiten Mitteilung ${ }^{1}$ ) sind wir auf die chemische Zusammensetzung der nach Bertrands Vorschrift dargestellten Medicago-Laccase eingegangen; sie erwies sich als eine Mischung von Calciumsalzen aliphatischer Oxysäuren, besonders Glykolsäure, Citronensäure, Äpfelsäure und Mesoxalsäure.

In Verfolgung der erwähnten Arbeiten sind wir der Frage näher getreten, ob die Laccasen hinsichtlich ihrer Wirksamkeit und Zusammensetzung einander so nahe stehen, daß sie in eine besondere Enzymgruppe zusammengefaßt werden können, und wir haben die Medicago-Laccase mit der zuerst bekannt gewordenen und typischen Laccase, derjenigen aus Rhus vernicifera verglichen.

Im Anschluß an eine Diskussion über die Rolle der Oxydasen im Pflanzenkörper ${ }^{2}$ ) und die Mitwirkung der Peroxydasen an vitalen Oxydationen haben wir eine Peroxydase näher untersucht. Wir berühren hier einen für die Systematik der Oxydasen grundlegenden Punkt. Abweichend von der Auffassung Bertrands, Bourquelots u. a. haben bekanntlich Chodat und Bach behauptet, daß "Oxydasen» Mischungen sind von Oxygenasen (eiweißartigen Körpern, welche den molekularen Sauerstoff unter Peroxydbildung aufnehmen) und Peroxydasen (Stoffen, welche das Oxydationsvermögen der Oxygenasen anderen Körpern gegenüber erhöhen).

Über die Berechtigung dieser Systematik und über den Enzymcharakter der Peroxydasen sind die Meinungen geteilt, je nach dem Material, mit welchem sich die einzelnen Autoren beschäftigt haben. ${ }^{3}$ ) Es schien uns deswegen wünschenswert, die Oxydationswirkungen von Präparaten verschiedener Herkunft quantitativ $\mathrm{zu}$ vergleichen.

$\mathrm{Zu}$ letzterem Zweck war es erforderlich, eine der typischen Peroxydase-Reaktionen zu einer quantitativen Messungen anwendbar zu machen. Es wurde hierzu die Guajakblaureaktion und die Rotfärbung von Guajakol gewählt, und wir beginnen damit, unsere Arbeitsweise $\mathrm{zu}$ beschreiben.

1) Diese Zeitschrift, Bd. LXI, S. 1 [1909].

2) Zeitschrift f. physikal. Chem., Arrhenius-Jubelband.

$\left.{ }^{3}\right)$ Ber. d. Deutsch. chem. Ges., Bd. XXXVI, S. 606 [1903]. 


\section{Kolorimetrische Bestimmung des Peroxydase-Gehaltes.}

Die angewandte Reaktion ist die bekannte Bildung eines blauen Oxydationsproduktes der Guajakonsäure nach Zusatz von $\mathrm{H}_{2} \mathrm{O}_{2}$ und eines Tier- oder Pflanzenstoffes, welchen wir wie üblich als "Peroxydase» bezeichnen. ${ }^{1}$ )

Es wurde nicht, wie dies bei der Anstellung der Guajakprobe bisher fast ausschließlich geschehen ist, das rohe Harz gelöst, sondern die Guajakonsäure wurde nach den Vorschriften von Doebner und Lücker ${ }^{2}$ ) aus dem Harz dargestellt ( $\alpha$ - und $\beta$ Säure wurden nicht getrennt); die Säure kam in $96 \%$ igem Alkohol gelöst in einer Konzentration von $0,1 \mathrm{~g}$ auf $100 \mathrm{ccm}$ Lösung zur Anwendung.

Die Versuche wurden in folgender Weise ausgeführt:

Es wurde $1 \mathrm{ccm}$ der zu prüfenden Lösung mit $1 \mathrm{ccm}$ einer $0,1 \%$ igen Wasserstoffsuperoxydlösung gemischt, worauf sie $\mathrm{zu}$ $2 \mathrm{ccm}$ Guajakonsäurelösung zugesetzt wurden. Diese Mischung geschah im Zylinder eines Gall enkampschen Kolorimeters. Als Vergleichslösung diente eine Lösung von Indigocarmin, deren Farbe am besten mit dem Guajakblau übereinstimmte. Es wurde nun unter Anwendung verschiedener Konzentrationen der peroxydasehaltigen Flüssigkeiten teils das Maximum der Absorption bestimmt, welche überhaupt eintritt, und zweitens diejenige Zeit, welche bis zur Erreichung der halben Farbenintensität verfließt.

Unter der meist, wenigstens angenähert, zutreffendenVoraussetzung, daß das Maximum der Absorption den Endzustand der Oxydation, also den vollständigen Verlauf der Reaktion kennzeichnet, wird, wenn die Absorption nach dem Beerschen Gesetz proportional der Konzentration des Guajakblaus ist, die Zeit, welche bis zum Ablauf der halben Reaktion verläuft, durch die Gleichung dargestellt:

1) Es soll gleich betont werden, daß wir mit dieser Bezeichnung nichts über die Enzymnatur, Einheitlichkeit oder Beschaffenheit dieses Stoffes aussagen.

2) Arch. d. Pharm., Bd. CCXXXIV, S. 590 (1896). 


$$
\begin{aligned}
0,4343 \mathrm{k} & =\frac{1}{\mathrm{t}} \log 2 \\
\text { also } \quad \mathrm{k} & =\frac{1}{\mathrm{t}} 0,693
\end{aligned}
$$

wenn $\mathrm{k}$ die Reaktionskonstante für Reaktionen 1. Ordnung bezeichnet.

Da es sich hier um die Oxydation der $\alpha$-Guajakonsäure durch $\mathrm{H}_{2} \mathrm{O}_{2}$ handelt, so wäre eigentlich die Geschwindigkeit nach der für bimolekulare Reaktionen gültigen Formel zu berechnen. Indessen befindet sich $\mathrm{H}_{2} \mathrm{O}_{2}$ in der Mischung in 10 fachem Überschuß, so daß seine Konzentration als praktisch konstant angesehen werden kann und die Reaktion monomolekular wird.

Über die Fehlergrenzen geben folgende Zahlen Aufschluß.

Das «Maximum» läßt sich im Kolorimeter mit einer mittleren Abweichung von 5\% bestimmen. (In Betracht kommen die Fehler bei der Verdünnung und der Ablesung an der Kolorimeterskala.) Die Dauer der halben Reaktion wurde mit einem Chronoskop auf $1 / 5$ Sekunden genau gemessen.

Folgende Tabelle zeigt die Beziehung, welche zwischen Konzentration und Reaktionsgeschwindigkeit herrscht.

$\begin{array}{lrrrr}\text { Verdünnung des peroxydasehaltigen Saftes } & 500 & 1000 & 2000 \\ \text { Maximum in Skalenteilen . . . . . . } & 80 & 66 & 52 \\ \text { Dauer der halben Reaktion in Sekunden } & 50 & 75 & 110 \\ 1000 \mathrm{k} \text {. . . . . . . . . . . . } & 14 & 9,3 & 7\end{array}$

Die Reaktionsgeschwindigkeit nimmt, wie ersichtlich, langsamer zu als die Konzentration, etwa proportional mit der Quadratwurzel derselben.

Eine passende Vergleichslösung, deren Farbe in einfacher Weise durch die Konzentration definiert werden könnte, haben wir nicht gefunden.

Einigermaßen reproduzierbar werden unsere Zahlen durch folgende Angaben, welche sich nicht auf Peroxydase, sondern auf Ghinon bezw. Ferrisulfat beziehen:

I. An Stelle der Peroxydaselösung wird $1 \mathrm{ccm}$ einer 0,01 normalen wässerigen Chinonlösung mit $1 \mathrm{ccm} 0,1$ normalem Wasserstoffsuperoxyd und mit $2 \mathrm{ccm}$ Guajakonsäurelösung versetzt. Das Absorptionsmaximum stellt sich nach etwa $10 \mathrm{Mi}-$ nuten ein. Die Absorption ist dann 1/s derjenigen der Ver- 
gleichslösung. (Nach Eintritt des Maximums geht die Farbenintensität der Guajakonsäurelösung wieder zurück, was den Wert der Bestimmung beeinträchtigt.)

Eine 0,1 normale Chinonlösung gab das Maximum 50; Proportionalität zwischen Chinonkonzentration und Menge des schließlich gebildeten Guajakblaus findet also nicht statt, eben wegen der Veränderlichkeit des gebildeten Farbstoffes in Gegenwart von Oxydationsmitteln.

II. Wenig bessere Resultate lieferte Ferrisulfat; es kam eine 0,0006 normale Lösung von Ferrosulfat zur Verwendung, welche durch ein gleiches Volumen von 0,1 normalem Wasserstoffsuperoxyd oxydiert worden war.

Sonstige Versuchsbedingungen wie bei I.

Absorptionsmaximum: 22 Skalenteile.

Ähnliche Versuche mit größeren Eisenkonzentrationen sind angestellt worden, um zu sehen, wie groß der Eisengehalt des Peroxydasepräparats sein müßte, um die Wirksamkeit dieser Präparate verursachen zu können. Es ergab sich, daß ein Präparat von der Wirksamkeit

\section{Maximum 100 Skalenteile.}

Dauer der halben Reaktionszeit 50 Sekunden wenigstens $50 \%$ Eisen enthalten müßte, wenn dieses allein der wirksame Bestandteil wäre. Tatsächlich enthalten die Präparate aus Meerrettichperoxydase nur Spuren von Eisen.

Der Saft aus frischen Kartoffeln, dessen Wirksamkeit S. 77 angegeben ist, enthielt in

$$
\begin{aligned}
800 \mathrm{ccm}: & 0,167 \mathrm{~g} \mathrm{Al} \\
0,446 & \gg \mathrm{Mg}
\end{aligned}
$$

dagegen nur Spuren von Eisen und Mangan.

Die Farbenreaktion mit Guajakol hat vor derjenigen mit Guajakonsäure den Vorzug, daß erstere Substanz leichter in reiner Form zu erhalten ist. Sie wurde auch schon früher von Bertrand zu quantitativen Messungen benutzt. Es war jedoch nicht möglich, wie bei Guajakonsäure, einen Endwert der Reaktion (ein Absorptionsmaximum) zu bestimmen, da nach längerer oder kürzerer Zeit stets das rote Oxydationsprodukt ausfällt, welches nach Bertrand Tetraguajakochinon ist. Wir mußten 
uns deshalb darauf beschränken, die Zeit zu bestimmen, welche bis zum Eintritt einer gewissen, willkürlich gewählten Farbe verfließt. Als Vergleichslösung diente eine ca. 2 normale Kaliumbichromatlösung, welche mit etwas saurer Lackmuslösung versetzt war. Die angewandte Guajakollösung war 0,05 normal. Der peroxydasehaltige Saft wurde im Verhältnis $1: 500,1: 1000$ und 1: 2000 verdünnt. Die weitere Mischung erfolgte wie früher: auf $1 \mathrm{ccm}$ der verdünnten Lösung kam $1 \mathrm{ccm} \mathrm{0,1} \mathrm{normalem}$ $\mathrm{H}_{2} \mathrm{O}_{2}$ und $2 \mathrm{ccm} 0,05$ normaler Guajakollösung.

Zwischen den Zeiten des Eintritts gleicher Färbung und der Konzentration der Peroxydase besteht folgende Abhängigkeit:

Verdünnung des peroxydasehaltigen Saftes: $500 \quad 1000 \quad 2000$

Zeit bis zum Eintritt der Vergleichsfarbe: $100 \quad 190 \quad 350$ Sekunden.

Die Proportionalität zwischen Reaktionszeit und Verdünnung ist also nur unvollkommen.

Es liegen in der Literatur keine Anhaltspunkte dafür vor, ob die Oxydation des Guajakols und der Guajakonsäure durch die gleichen Stoffe bezw. die gleiche Peroxydase bewirkt wird. Um darüber einen Aufschluß zu gewinnen, wurde untersucht, ob bei verschiedenen Pflanzenpräparaten und Säften die Oxydation dieser beiden Substanzen parallel geht. Zur Untersuchung kamen die Lösung eines Peroxydasepräparates aus Meerrettich (0,01 $\mathbf{g}$ in 200 Wasser) (A) ferner die aus Äpfeln (B) und aus Kartoffeln (C) durch Auspressen gewonnenen Säfte, erstere unverdünnt.

Rotfärbung des Guajakols: A B C (verdünnt 1:20)

Eintritt der Vergleichsfarbe nach $220 \quad 300 \quad 85$ Sekunden

Bildung von Guajakblau:

$\begin{array}{lllr}\text { Maximum . . . . . . . . } & 60 & 17 & 40 \\ \text { Dauer der halben Reaktion . . } & 85 & - & 120\end{array}$

Zwischen den beiden Peroxydasereaktionen, der Bildung des Tetraguajakochinons und des Guajakblaus, besteht also keine Parallelität, und es ist also anzunehmen, daß die beiden Reaktionen durch verschiedene Stoffe katalysiert werden. 
Die Abhängigkeit der Guajakblaureaktion von Säuren, Alkalien und einigen Giften.

$20 \mathrm{ccm}$ einer 10\% 10 igen Lösung von Rohperoxydase aus Meerrettich wurden mit 2,5 ccm einer 0,2 normalen Salzsäurelösung ${ }^{1 / 2}$ Stunde stehen gelassen. Hierauf wurde mit $2,5 \mathrm{ccm}$ einer 0,2 normalen Natronlauge neutralisiert; nach Verdünnung dieser Lösung im Verhältnis $1: 500$ wurde dann wie gewöhnlich Maximum und Geschwindigkeit der eintretenden Blaufärbung bestimmt.

Gefunden: Maximum 100;

Dauer der halben Reaktion 20 Sekunden.

Ein Parallelversuch, bei welchem an Stelle der Salzsäure ein gleiches Volumen Wasser angewandt wurde, gab:

Maximum 100; Dauer der halben Reaktion 25 Sekunden.

$10 \mathrm{ccm}$ einer $10 \%$ igen Lösung von Rohperoxydase wurden mit 2,5 ccm einer 0,2 normalen Natronlauge $3 / 4$ Stunden lang stehen gelassen. Dann wurden $2,5 \mathrm{ccm} 0,2$ normaler Salzsäure zugesetzt; nach Verdünnung der Lösung von 1 auf 200 ergab sich:

Maximum 100; Dauer der halben Reaktion 50 Sekunden.

Parallelversuch mit Wasser:

Maximum 100; Dauer der halben Reaktion 51.

Es zeigt sich also, daß die Wirksamkeit der Peroxydase durch geringe Mengen Säuren und Basen (in rund 0,02 normaler Lösung) nicht geändert wird.

Wir haben im Anschluß hieran auch den Einfluß der Acidität auf die Guajakblaufärbung untersucht, also Peroxydase, Wasserstoffsuperoxyd und Guajakonsäure in saurer, neutraler und in alkalischer Lösung gemischt, besonders um zu erfahren, $o b$ und wie durch den zufälligen Säuregehalt natürlicher Pflanzensäfte die Guajakblaureaktion beeinträchtigt wird. Dabei hat sich gezeigt, daß mit freiem Auge Unterschiede in der Guajakblaureaktion nicht wahrgenommen werden können, zwischen Lösungen, welche 0,01 normal in bezug auf Salzsäure und Lösungen, welche 0,01 normal in bezug auf $\mathrm{NaOH}$ sind.

Im Anschluß an die Versuche über den Einfluß von Säuren und Basen haben wir auch den Einfluß einiger Gifte untersucht. 
Über die Wirkung von Giften auf Pilzoxydasen hat Kastle bemerkenswerte Beobachtungen mit.geteilt. ${ }^{1}$ ) Energisch und dauernd geschädigt wurden die Pilzoxydasen besonders durch Chloroform, ferner durch Thymol, während sie gegen Formaldehyd sehr unempfindlich sind. Starke Hemmungen erzeugen nach Laqueur ${ }^{2}$ ) und nach Rosenfeld ${ }^{3}$ ) die Alkaloide, besonders Chinin.

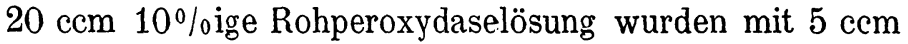
$1 \%$ iger Formaldehydlösung versetzt. Verdünnung im Verhältnis 1: 500 wie bei dem Versuch mit Salzsäure.

Die Blaufärbung mit der so verdünnten Lösung wurde bestimmt:

1. Unmittelbar nach der Mischung.

2. Nach einer halben Stunde.

3. Nach 24 Stunden.

Alle drei Versuche und ein Parallelversuch mit $5 \mathrm{ccm}$ Wasser ergaben das übereinstimmende Resultat:

Maximum 100; Dauer der halben Reaktion: 25 Sekunden.

Ebensowenig Einfluß wie der Formaldehyd hatten Zusätze von $5 \mathrm{ccm}$ folgender Lösungen:

a) 0,005 g Thymol, aufgeschlämmt in Wasser,

b) $0,05 \mathrm{~g}$ gelöst in sehr verdünntem wässerigem Alkohol,

c) gesättigte, wässerige Chloroformlösung,

d) $0,1 \%$ ige Chininsulfatlösung.

Wenn auch die Fehlergrenzen der beschriebenen kolorimetrischen Bestimmungsarten erheblich größer sind, als man gewöhnlich an analytische Methoden stellt, so gestatten sie immerhin in einfacher Weise den Gehalt an "Peroxydase " mit einern mittleren Fehler von etwa $10 \%$ zu ermitteln, und die quantitativen Beobachtungen sind, wenn es sich um Feststellung und Vergleich von Peroxydasegehalten verschiedener Pflanzenteile und Präparate handelt, den bisher üblichen qualitativen Reagenzglasversuchen entschieden vorzuziehen. Wir haben die verschiedenen Fraktionen bei den Reinigungsver-

1) Chemisches Zentralblatt, Bd. LXXVII, 1, S. 1555 (1906).

2) Arch. f. exp. Path., Bd. LV, S. 240 (1906).

3) Dissertation, Petersburg 1906. 
suchen mit Cochlearia-Peroxydase auch stets in der oben angegebenen Weise untersucht.

\section{Reinigungsversuche mit Peroxydase aus Meerrettich (Cochlearia armoracia).}

Es bestätigte sich zunächst, daß Meerrettichwurzeln hervorragend kräftige Peroxydasereaktionen liefern und insofern als Ausgangsmaterial für die Reindarstellung in erster Linie in Betracht kommen. Bach und Chodat ${ }^{1}$ ) haben die CochleariaPeroxydase $\mathrm{zu}$ isolieren versucht, und gelangten $\mathrm{zu}$ einem Präparat von folgenden Eigenschaften: «Gelblichweiße, gummiartige Masse, in Wasser außerordentlich löslich, in $40 \%$ jgem Alkohol leicht löslich. Reduziert stark Fehlings Lösung. Die reinsten Präparate enthalten durchschnittlich $6 \%$ Asche. Mehrere Analysen ergaben, daß dieselbe eisenfrei, dagegen aluminium- und manganhaltig ist. Der Gehalt an Aluminium beträgt $0,8-1,4 \%$, derjenige an Mangan $0,2-0,6 \%$. $O b$ $z$ wischen dem Mangangehalt und den Peroxydaseeigenschaften des Produkts ein kausaler Zusammenhang besteht, konnte mit Sicherheit nicht nachgewiesen werden. Beim Erwärmen von Peroxydaselösungen mit Natronlauge entweicht zuerzt Ammoniak und dann eine nach Pyridin riechende Base.»

Nach einer Feststellung von Tschirch und Stevens, ${ }^{2}$ ) daß sich aus Rhus-Laccase bei der Destillation mit Natronkalk Pyrrol entwickelt, wies A. $\mathrm{Bach}^{3}$ ) nach, daß Meerrettichoxydasen bei der Destillation mit Kali Ammoniak und Pyrrol liefern.

Schließlich ist eine eingehende Arbeit von E. de Stoeck lin ${ }^{4}$ ) aus Chodats Laboratorium zu erwähnen, welche ebenfalls die Peroxydase aus Cochlearia armoracia betrifft. Nach Stoecklin ist diese Substanz amorph, aber kein Eiweiß. Die sie begleitenden anorganischen Bestandteile sind besonders Phosphate

1) Ber. d. Deutsch. chem. Ges., Bd. XXXVI, S. 600 (1903).

2) Arch. d. Pharm., Bd. GCXLIII (7), S. 504 (1905).

s) Ber. d. Deutsch. chem. Ges., Bd. XLI, S. 226 (1908).

4) Inst. bot. de l'Univ. de Genève, sér. 7, S. 39 (1907). 
des $\mathrm{Ca}, \mathrm{Mg}, \mathrm{Na}$ und $\mathrm{K}$. Auch stickstoffhaltige Substanzen finden sich in der Peroxydase. Besonders wird die Abwesenheit von Mangan in der Meerrettichperoxydase betont.

Sowohl die von Bach und Chodat als die von Stoecklin schließlich erhaltenen Präparate haben offenbar noch viel inaktive, nicht zur Peroxydase gehörende Substanzen enthalten. Wir haben deswegen versucht, ob durch systematisches Fraktionieren oder Dialysieren eine weitere Reinigung möglich ist.

I.

$6 \mathrm{~kg}$ Meerrettich wurden fein gerieben und abgepreßt. ${ }^{1}$ ) Es wurden 3 Liter Saft gewonnen, aus welchem sich in wenigen Stunden reichliche Mengen Stärke und andere feste Substanzen abscheiden. Der von der Fällung dekantierte Saft wird mit Baryt neutralisiert, um die Hauptmasse der Phosphorsäure zu entfernen; der Rest der Phosphorsäure wurde durch Behandeln des Saftes mit Baryumcarbonat entfernt. Ohne vorhergehende Filtration wurde soviel $96 \%$ iger Alkohol zugesetzt, daß die Lösung darn $33 \%$ Alkohol enthielt. Die Fällung wird abgesaugt. Ein Teil des Filtrates wurde mit Tierkohle behandelt; sowohl dieser Teil als der Rest wird filtriert; das Filtrat wird mit dem 5-6fachen Volumen Aceton versetzt, wodurch die Peroxydase mit einem breiigen, zähen Sirup ausfällt. Diese Fällung wird in möglichst wenig Wasser gelöst und in viel absoluten Alkohol eingegossen, wobei nun weiße Flocken ausfallen, welche zweckmäßig mit Alkohol zerrieben und mit Äther gewaschen werden.

Nach Absaugen wurden $162 \mathrm{~g}$ eines trockenen, gelblichen

1) Wird die zerriebene und ausgepreßte Masse direkt mit kaltem, $40 \%$ igem Alkohol extrahiert, so löst sich nur wenig Substanz. Die Lösung wurde mit Baryt neutralisiert, die Fällung wurde nach einiger Zeit abfiltriert. Durch Fällen des wässerig-alkoholischen Filtrates wurden $8 \mathrm{~g}$ Substanz exhalien. $5 \mathrm{~g}$ davon wurden während 48 Stunden durch Pergamentfilter dialysiert. Der Hülseninhalt wird mit absolutem Alkohol gefällt. Es werden so $0,3 \mathrm{~g}$ einer rein weißen Substanz erhalten, welche $8,4 \%$ Asche enthält; die Guajakprobe fällt folgendermaßen aus (siehe S. 74):

Maximum: 88. Dauer der halben Reaktion: 50 Sekunden.

Hoppe-Seyler's Zeitgchrift f. physiol. Chemie. LXI. 
Pulvers erhalten. Ein sehr großer Teil dieses Präparates besteht aus Rohrzucker und Glukose, welch letztere (ohne vorhergehende Inversion durch Säuren) als Osazon ausgefällt wurde.

Der Peroxydasegehalt ergibt sich aus folgender Messung mit Guajakonsäure. Hier wie bei allen Versuchen 0,01 g Enzympräparat in $200 \mathrm{ccm}$ Wasser. Von dieser Verdünnung wurde, wie S. 74 angegeben, $1 \mathrm{ccm}$ mit $1 \mathrm{ccm} \mathrm{H}_{2} \mathrm{O}_{2}$-Lösung und $2 \mathrm{ccm}$ Guajakonsäurelösung gemischt.

Maximum: 70; Dauer der halben Reaktion: 90 Sekunden.

$20 \mathrm{~g}$ dieses Präparates $\mathrm{P}_{1}$ werden während 48 Stunden durch Diffusionshülsen von Schleicher und Schüll Nr. 579 gegen fließendes Wasser dialysiert. Es zeigte sich durch besondere Versuche, daß dabei keine Peroxydase durch diese Hülsen diffundiert. ${ }^{1}$ ) Während der Dialyse fallen Eiweißkörper aus, von welchen abfiltriert wurde. Nach der genannten Zeit wird der Inhalt der Hülsen in einen großen Ü̉berschuß von absolutem Alkohol eingetragen, wodurch 1,5 g einer rein weißen Fällung $M$ erhalten wird.

Peroxydasegehalt:

Maximum: 100; Dauer der halben Reaktion: 23 Sekunden.

Eine Aschenbestimmung der bei $80^{\circ}$ getrockneten Substanz lieferte 7,37\% Asche.

0,1873 g Substanz gaben beim Glühen $0,0138 \mathrm{~g}$ Rückstand.

Der Rückstand von $\mathrm{M}, 1,3 \mathrm{~g}$ betragend, wird wieder in wenig Wasser gelöst und während 72 Stunden dialysiert. Hierauf wird mit absolutem Alkohol gefällt, wodurch $0,21 \mathrm{~g}$ Substanz erhalten werden.

Gefunden: Maximum: 100; Dauer der halben Reaktion:7 Sekunden.

Durch die fortgesetzte Dialyse wurde somit die Wirksamkeit des Präparates, und wie man wohl annehmen darf, der Peroxydasegehalt bedeutend vergrößert.

1) Im Gegensatz zu dem Versuch ron Bach und Ghodat (Ber. der Deutsch. chem. Ges., Bd. XXXVI, S. 602), welche ein peroxydasehaltiges Dialysat erhalten haben. 
Eine Aschenbestimmung der bei $80^{\circ}$ getrockneten Substanz lieferte 2,67\% Asche.

0,1831 g Substanz gaben beim Glühen 0,0048 g Rückstand.

II.

$50 \mathrm{~g}$ eines Präparates, welches ganz analog wie $\mathrm{P}_{1}$ gewonnen worden, aber nicht mit Tierkohle behandelt worden war $\left(\mathrm{P}_{1 \mathrm{a}}\right)$, wurde gegen fließendes Wasser dialysiert. Nach 72 Stunden wurde die Dialyse unterbrochen, um von den ausgeschiedenen Eiweißkörpern abzufiltrieren. Nach weiteren 96 Stunden wurde der Hülseninhalt mit großem Überschuß von Alkohol gefällt, wodurch $0,72 \mathrm{~g}$ eines gelblich-braunen Pulvers erhalten wurden.

Wirksamkeit dieses Präparates:

Maximum: 100; Dauer der halben Reaktion: 10 Sekunden.

Das Präparat war also annähernd ebenso wirksam wie das während 120 Stunden dialysierte Präparat $P_{1}$ (S. 82).

Eine Aschenbestimmung der bei $80^{\circ}$ getrockneten Substanz lieferte 2,49\% Asche.

$0,1845 \mathrm{~g}$ Substanz gaben $0,0046 \mathrm{~g}$ Rückstand.

Eine Stickstoff bestimmung lieferte $10,4 \% \mathrm{~N}$.

0,1902 g Substanz gaben $17,29 \mathrm{ccm} \mathrm{N} \mathrm{N}_{8}\left(18^{\circ}\right.$ und $\left.757 \mathrm{~mm}\right)$.

An einem genau wie $P_{1}$ erhaltenen Präparat $P_{2}$ wurde untersucht, ob durch Fraktionierung mit Alkohol eine Anreicherung der Peroxydase erzielt werden kann.

Wirksamkeit von $\mathrm{P}_{q}$ :

Maximum: 38; das Präparat war also schwächer als $P_{1}$.

a) $5 \mathrm{~g}$, in $100 \mathrm{ccm} 50 \%$ igem Alkohol aufgeschlämmt, bildeten eine feine Emulsion, aus welcher sich über Nacht ein Niederschlag absetzte. Derselbe wies nach Absaugen und Waschen mit Alkohol folgende Wirksamkeit:

Maximum: 60. Die Wirksamkeit hat sich also etwa verdoppelt.

b) $5 \mathrm{~g}$ wurden in $100 \mathrm{ccm} 70 \%$ igem Alkohol aufgeschlämmt. Die Substanz geht dabei auffallenderweise fast vollständig in Lösung. Durch Fällen mit absolutem Alkohol 
wird ein weißes Präparat erhalten, welches gegenüber Guajakonsäure nur eine außerordentlich geringe Wirksamkeit zeigt (Maximum etwa $0-5$ ).

Eine wirksame Reinigungs- bezw. Anreicherungsmethode läßt sich also auf die fraktionierte Fällung durch Alkohol nicht gründen.

Am Präparat $\mathrm{P}_{2}$ wurde der Zuckergehalt nachgewiesen. Aus $2 \mathrm{~g}$ Substanz wurden zunächst die Eiweißkörper durch Kochen entfernt, worauf eine Stunde mit essigsaurem Phenylhydrazin im Wasserbade erhitzt wurde. Nach dem Abkühlen wurden 0,2 g Osazon erhalten, welches nach einmaligem Umkrystallisieren aus Alkohol bei $201-202^{\circ} \mathrm{schmolz}$, also zweifellos Glukosazon war.

Um zu erfahren, ob der in unseren verschiedenen Präparaten gefundene Stickstoffgehalt von Proteinen herrührt, wurden $50 \mathrm{~g}$ von $\mathrm{P}_{2}$ folgender Behandlung unterworfen:

Es wurde mit 7-tägiger Dialyse begonnen. Der Hülseninhalt wird in zwei Hälften geteilt. Die eine Hälfte wurde mit Tierkohle gekocht, filtriert und mit absolutem Alkohol gefällt.

Die so erhaltene, bei $80^{\circ}$ getrocknete Substanz wurde zur Untersuchung des Stickstoff- und Aschengehaltes verwendet. Die Substanz enthielt: 7,47\% N und 8,02\% Asche.

Stickstoffbestimmung:

$0,2074 \mathrm{~g}$ Substanz gaben $13,46 \mathrm{ccm} \mathrm{N}_{\mathbf{2}}\left(17^{\circ}\right.$ und $\left.758 \mathrm{~mm}\right)$.

Aschenbestimmung:

0,2061 g Substanz gaben 0,0407 g Rückstand.

III.

$6 \mathrm{~kg}$ Meerrettich, gerieben und abgepreßt, lieferten $3250 \mathrm{ccm}$ Saft. Derselbe wurde so rasch als möglich in flachen Porzellanschalen auf freier Flamme bis zur Koagulation der Eiweißstoffe erhitzt. Nach etwa 5 Minuten wurde schnell in Eiswasser abgekühlt. Durch diese Behandlung wird die Peroxydase-Wirkung des Saftes zwar geschwächt, aber bei weitem nicht aufgehoben, wie folgende mit einem im Verhältnis 15 : 500 verdünnten Saft angestellten Versuche zeigen: 


$\begin{array}{lcc} & \text { Maximum } & \text { Dauer } \text { der halben Reaktion } \\ \text { Ungekocht } & 100 & 21 \text { Sekunden } \\ \text { Gekocht } & 85 & 50\end{array}$

Ohne von den Eiweißkörpern abzufiltrieren, wird der gekochte Saft mit Baryt neutralisiert, mit Baryumcarbonat digeriert und mit soviel Alkohol versetzt, daß die Lösung 30\%ig wurde. Es wurde nun abgesaugt, das Filtrat mit Aceton gefällt, der Niederschlag in Wasser gelöst und mit absolutem Alkohol gefällt. Wir erhielten von diesem Präparat $\mathrm{P}_{3} 137 \mathrm{~g}$.

Wirksamkeit des Präparates $\mathrm{P}_{3}$ gegen Guajakonsäure: Maximum: 38; Dauer der halben Reaktion: 140 Sekunden.

$50 \mathrm{~g}$ des Präparates $\mathrm{P}_{3}$ werden während 7 Tagen unter Austausch der Hülsen dialysiert. Der Hülseninhalt wird wie früher mit absolutem Alkohol gefällt und gewaschen; erhalten werden $3,6 \mathrm{~g}$ dieses Präparates $\mathrm{p}_{3}$.

Wirksamkeit gegen Guajakonsäure:

Maximum: 88; Dauer der halben Reaktion: 55-60 Sekunden.

Analysen ergaben 7,5\% Asche und 7,77\% N.

$0,1775 \mathrm{~g}$ Substanz lieferten $0,0135 \mathrm{~g}$ Rückstand.

0,2103 > > $14,03 \mathrm{ccm} \mathrm{N}_{2}\left(17^{\circ}\right.$ und $\left.766,5 \mathrm{~mm}\right)$.

Von diesem Präparat $\mathrm{p}_{3}$ werden etwa $2 \mathrm{~g}$ mit wenig Wasser behandelt; die Lösung wurde gekocht und ein sich dabei bildender Niederschlag nun abfiltriert. Der Niederschlag wurde auf seinen Stickstoffgehalt untersucht; derselbe betrug $4,79 \%$.

$0,1601 \mathrm{~g}$ bei $80^{\circ}$ getrocknete Substanz gaben $6,05 \mathrm{ccm} \mathrm{N}_{2}$ $\left(18^{\circ}\right.$ und $\left.762 \mathrm{~mm}\right)$.

Der beim Kochen gelöst gebliebene Teil von $p_{3}$ wird mit 'Tierkohle gekocht, abfiltriert und mit absolutem Alkohol gefällt. Die bei $80^{\circ}$ getrocknete Substanz enthielt $4,12 \% \mathrm{~N}$.

$0,2507 \mathrm{~g}$ Substanz gaben $8,92 \mathrm{ccm} \mathrm{N}_{2}\left(17^{\circ}\right.$ und $\left.762 \mathrm{~mm}\right)$.

Weitere $50 \mathrm{~g}$ von $\mathrm{P}_{3}$ werden, wie oben erwähnt, dialysiert, dieses Mal in weiten Hülsen. Nach 7 Tagen (nach 3 Tagen wurden die Hülsen gewechselt) wurden aus dem Hülseninhalt durch Alkohol 5,5 g ausgefällt.

Wirksamkeit dieses Präparates $\pi_{3}$ :

Maximum: 60; Dauer der halben Reaktion: 85 Sekunden.

Die Wirksamkeit war also bei diesem Präparat schwächer. 
Durch die weiteren Hülsen waren in den 7 Tagen weniger unwirksame Substanzen herausdialysiert (in ersterem Versuch wurden 3,6 , in letzterem $5,5 \mathrm{~g}$ Substanz erhalten). Je länger also die Dialyse getrieben wird, desto wirksamer wird die Substanz.

Die Analyse von $\pi_{3}$ ergab 6,61\% Asche. 0,1694 g Substanz gaben 0,0112 g Rückstand.

$3 \mathrm{~g}$ des Präparates $\pi_{3}$ werden vollständig gelöst, mit Tierkohle gekocht; nach Absaugen des eintretenden Niederschlages wird mit absolutem Alkohol gefällt, die erhaltenen $0,25 \mathrm{~g}$ werden bei $80^{\circ}$ getrocknet. Sie enthielten $11,27 \%$ Asche und $4,16 \% \mathrm{~N}$.

0,1100 g Substanz gaben 0,0124 g Rückstand.

0,1090 > $\quad 3,81 \mathrm{~cm} \mathrm{~N} \mathrm{~cm}_{2}\left(15,5^{\circ}\right.$ und $\left.778 \mathrm{~mm}\right)$.

$1,6595 \mathrm{~g}$ des Präparates $\pi_{3}$ wurden in $30 \mathrm{ccm} 2 \%$ iger Natronlauge und etwas Wasser gelöst und der Destillation unterworfen, wobei flüchtige Basen quantitativ in titrierter Salzsäure aufgefangen wurden. Nach Übergang von $50 \mathrm{ccm}$ Destillat wurde von neuem Wasser zugesetzt und die Destillation fortgesetzt. Durch die übergehenden Basen wurden $7,5 \mathrm{ccm}$ 0,2 normaler Salzsäure neutralisiert; es waren somit 0,0015 g-Moleküle einer Base übergegangen. Diese wird einer näheren Untersuchung unterworfen.

Die im Vorhergehenden angeführten Daten können in folgender Tabelle zusammengefaßt werden, um den Einfluß der Dialyse auf Stickstoff- und Aschengehalt und Wirksamkeit zu zeigen.

Die Dialyse verläuft folgendermaßen: Zuerst verliert die Lösung hauptsächlich Zucker, so daß der Aschengehalt steigt; im weiteren Verlauf der Dialyse entfernen sich verhältnismäßig mehr Mineralstoffe, deren Gehalt in den durch Alkohol gefällten Präparaten also sinkt, während der Peroxydasegehalt (gemessen durch die Wirksamkeit gegenüber Guajakonsäure) zunimmt. Die an zwei verschiedenen Präparaten, $\mathrm{P}_{\mathbf{1}}$ und $\mathrm{P}_{1 \mathrm{a}}$ (Zeile 3 und 4 der Tabelle) erhaltenen übereinstimmenden Zahlen scheinen bemerkenswert. 


\begin{tabular}{|c|c|c|c|c|c|c|c|c|}
\hline \multicolumn{4}{|c|}{ Präparat } & $\begin{array}{c}\text { Aschen- } \\
\text { gehalt } \\
\%\end{array}$ & $\begin{array}{l}\text { Stick- } \\
\text { stoff- } \\
\text { gehalt } \\
0 / 0\end{array}$ & $\begin{array}{r}\text { Guaja } \\
\text { Maximum }\end{array}$ & $\begin{array}{r}\text { kblaubi } \\
\text { Dau } \\
\text { halben }\end{array}$ & $\begin{array}{l}\text { ng } \\
\text { der } \\
\text { aktion }\end{array}$ \\
\hline 1. & $P_{1}$ nich & \multicolumn{2}{|c|}{ dialysiert } & - & - & 70 & \multicolumn{2}{|c|}{90 Sekunden } \\
\hline 2. & \multicolumn{2}{|c|}{48 Stund. } & $\triangleright$ & 7,4 & - & 100 & 23 & $\triangleright$ \\
\hline 3. & . 120 & , & D & 2,6 & - & 100 & 7 & $\triangleright$ \\
\hline 4. & $P_{1 a} 168$ & , & . & 2,5 & 10,4 & 100 & 10 & $\triangleright$ \\
\hline 5. & $\mathrm{P}_{3}$ dial & iert ${ }^{1}$ & . . & - & - & 38 & 140 & $\gg$ \\
\hline 6. & > $168 \mathrm{~S}$ & $\begin{array}{l}\text { nd.d } \\
\text { ne Hi }\end{array}$ & $\begin{array}{l}\text { lysiert } \\
\text { en) }\end{array}$ & 7,6 & 7,8 & 88 & $55-60$ & $\triangleright$ \\
\hline 7. & $\begin{array}{r}168 \mathrm{~S} \\
\text { (gr }\end{array}$ & $\begin{array}{l}\text { d. d } \\
\text { Hü }\end{array}$ & $\begin{array}{l}\text { lysiert } \\
\text { en) }\end{array}$ & 6,6 & - & 60 & 85 & $\triangleright$ \\
\hline
\end{tabular}

Geht man von sehr großen Mengen Rohmaterial aus und dialysiert lange, etwa mehrere Wochen, so kommt man wohl zu Präparaten von noch etwas höherem Stickstoffgehalt, höherer Wirksamkeit und geringerem Aschengehalt. Indessen werden solche Versuche sehr kostspielig und zeitraubend, und es wird sich eher empfehlen, ein anderes, von vornherein etwas reineres Ausgangsmaterial $\mathrm{zu}$ suchen.

Einfluß des Erwärmens auf den Saft und das mit Alkohol gefällte Präparat.

$10 \mathrm{ccm}$ Saft werden mit 90 ccmWasser gemischt (Versuche $\alpha$ ).

$5 \mathrm{~g}$ des Präparates $\mathrm{P}_{1}$ werden in $100 \mathrm{ccm}$ Wasser gelöst (Versuche $\beta$ ).

Diese beiden Lösungen werden in bezug auf ihre Wärmeempfindlichkeit verglichen. Unmittelbar nach Herstellung der Lösung wurden folgende Intensitäten gefunden, wobei beide Lösungen im Verhältnis 1:100 verdünnt waren:

a) Maximum: 100; Dauer der halben Reaktion: 38 Sekunden.

ß) Maximum: 100; Dauer der halben Reaktion; 25 Sekunden.

In ähnlicher Weise wurden die beiden Lösungen unter-

1) Dieses Präparat war durch Erhitzen der Lösung von Eiweiß stoffen zum größten Teil befreit worden. Die Erhitzung führte natürlich eine Schwächung des Präparates herbei. 
sucht, nachdem sie während 15, 45 und 115 Sekunden auf $100^{\circ}$ erwärmt waren. Die Maxima betrugen durchweg 100. Berechnet man die Geschwindigkeit, mit welcher die Peroxydase in der Wärme zersetzt wird, aus den Zeiten $t$ Sekunden für die halbe Guajakblaufärbung und aus den angewandten Verdünnungen $\mathrm{v}$, so erhält man folgende Übersicht über die Wirksamkeit der Lösungen, dargestellt durch die Werte tv:

\begin{tabular}{|c|c|c|c|c|}
\hline Dauer der Erwärmung in Sekunden . : . & 0 & 15 & $4 \check{5}$ & 115 \\
\hline Lösung $\alpha . . . . . . .$. & 38 & 110 & 600 & 1500 \\
\hline Lösung $\beta \cdot \cdot \cdot \cdot \cdot \cdot \cdot \cdot \cdot$ & 25 & 74 & 300 & - \\
\hline
\end{tabular}

Die Empfindlichkeiten des rohen Saftes und eines gelösten Peroxydasepräparates sind also nicht sehr verschieden. In beiden Fällen sinkt anfangs die Aktivität der Peroxydase außerordentlich stark.

Zur genaueren Bestimmung der Wärmeempfindlichkeit der Peroxydase müssen ähnliche Messungen bei etwa $80^{\circ}$ angestellt werden, wo die Erwärmungszeiten etwas länger gewählt werden können.

Von der Verdünnung scheint die Wärmeempfindlichkeit der Peroxydase unabhängig zu sein. Die gleiche Erhitzungsdauer schwächt die Peroxydase, wie die folgenden Versuche zeigen, in der gleichen Weise, wenn die Verdünnung auch im Verhältnis $1: 5$ varriert wird.

$5 \mathrm{~g}$ des Präparates $\mathrm{P}_{3}$ wurden in 50 ccm gelöst (Lösung $\mathrm{M}$ ).

1. Verdünnung $1: 200$. Ungekocht.

Maximum: 100; Dauer der halben Reaktion 25 Sekunden.

2. Nach Erhitzen der Lösung M während 100 Sekunden im kochenden Wasserbad wurde im Verhältnis 1:5 verdünnt.

Maximum: 100: Dauer der halben Reaktion 25 Sekunden.

3. Eine $5 \mathrm{mal}$ so verdünnte Lösung wie $\mathbf{M}$ wird gekocht (um den Einfluß der Verdünnung zu sehen), wird während 100 Sekunden erhitzt und unverdünnt untersucht:

Maximum: 100; Dauer der halben Reaktion 25 Sekunden. 


\section{Versuche mit Laccase aus Rhus vernicifera.}

Das Rohpräparat, für welches wir Herrn Professor H. Yoshida zu großem Dank verpflichtet sind, war bereits in Japan von mechanischen Verunreinigungen befreit worden und stellte eine ziemlich zähflüssige, weißliche Masse dar. (Es erweist sich stark wirksam als Oxydationskatalysator: Es färbt eine alkoholische Guajakonsäurelösung direkt (ohne $\mathrm{H}_{2} \mathrm{O}_{2}$ ) blau, Guajakollösungen rot.) Die Masse wird in sehr großem Überschuß $96 \%$ igen Alkohols unter starkem Umrühren eingegossen, vor dem Fällen dekantiert und die Fällung nochmals mit der gleichen Alkoholmenge behandelt. Der nun erhaltene Niederschlag wird abgesaugt, in wenig Wasser gelöst, und mit Alkohol wiederum gefällt.

Wir haben dieses Präparat zunächst dazu verwendet, die Wirkung der Rhus-Laccase auf Hydrochinon zu untersuchen, um somit dieses Präparat mit der Medicago-Laccase und mit alkalischen Manganlösungen $\mathrm{zu}$ vergleichen.

$0,2 \mathrm{~g}$ Laccase in $10 \mathrm{ccm}$ Wasser gelöst. Der an anderer Stelle $^{1}$ ) beschriebene Apparat, welchen wir auch bei unserer ersten Untersuchung angewandt hatten, wurde mit $20 \mathrm{ccm}$ einer 0,5 normalen Hydrochinonlösung und $20 \mathrm{ccm}$ destilliertem Wasser, hierauf mit Sauerstoff gefüllt und schließlich die $10 \mathrm{ccm}$ der Laccaselösung zugesetzt. Die Versuchsanordnung ist in unserer erwähnten Mitteilung zu ersehen.

I.

$\begin{array}{cc}\text { Minuten } & \text { ccm absorb. } \mathrm{O}_{2} \\ 3 & 1,2 \\ 5 & 2,8 \\ 10 & 5,1 \\ 15 & 8 \text { Ausfallen von } \\ 20 & 11,6\end{array}$

II.

$\begin{array}{cc}\text { Minuten } & \mathrm{ccm} \text { absorb. } \mathrm{O}_{8} \\ 3 & 1,4 \\ 5 & 2,5 \\ 10 & 5,4 \\ 15 & 9,0 \\ 20 & 12,0\end{array}$

Durch 3 Minuten langes Kochen wird die Wirksamkeit der Rhus-Laccase total aufgehoben.

1) Diese Zeitschrift, Bd. LVII, S. 80 (1908), und Zeitschrift für physikal. Chemie, Arrhenius-Jubelband. 
In diesem Zusammenhang sei hervorgehoben, daß die wässerige Lösung unseres Rhuspräparates sowohl gegen Lackmus als gegen Methylorange vollkommen neutral war. Es kann also keine Rede davon sein, daß die katalytische Wirkung der RhusLaccase einfach auf die Gegenwart von Mangan und von Hydroxylionen zurückgeführt werden kann.

Durch Behandlung der gelösten Rhus-Laccase mit Tierkohle wird der katalytische Effekt vermindert, wie folgender mit den obigen direkt vergleichbare Versuch ergibt:

III.

$\begin{array}{cc}\text { Minuten } & \text { ccm absorb. } \mathrm{O}_{2} \\ \mathbf{3} & 0,5 \\ \mathbf{5} & 0,7 \\ 10 & 1,4 \\ 15 & 3,1 \\ 20 & 4,3 \\ 30 & 5,7\end{array}$

Die mit Tierkohle behandelte Laccaselösung wird während 8 Tagen gegen fließendes Wasser dialysiert, worauf der Hülseninhalt mit Alkohol gefällt wird.

$0,2 \mathrm{~g}$ des so gewonnenen Präparates werden wie oben untersucht.

IV.

$\begin{array}{cc}\text { Minuten } & \text { ccm absorb. } \mathrm{O}_{\mathbf{s}} \\ 3 & 1,0 \\ 5 & 2,2 \\ 10 & 4,4 \\ 15 & 5,9 \\ 20 & 6,0\end{array}$

Die Rhus-Laccase unterscheidet sich demnach durch ihre Eigenschaften sehr stark von der Medicago-Laccase.

Während letztere ganz unempfindlich gegen Erhitzen ist, wird die Rhus-Laccase bei $100^{\circ}$ schnell getötet.

Das Präparat aus Rhus, nicht das aus Medicago wird durch Behandeln mit Tierkohle geschwächt.

Die Rhuspräparate, nicht aber die Medicago-Laccasen bläuen Guajakonsäurelösungen direkt und färben Guajakollösungen rot. 
Schließlich wurden ein paar Aschebestimmungen vorgenommen und zwar an dem direkt gefällten Präparat und an dem durch Dialyse gereinigten. Erstere Bestimmung lieferte $4,05 \%$ Asche, letztere 3,1\%. Bertrand gibt für eine seiner LaccaseFraktionen aus Rhus einen Aschengehalt von 5,17\% an.

\section{Zusammenfassung.}

I.

1. Bei Wahl geeigneter Konzentrationen von Guajakonsäure und Hydroperoxyd läßt sich mit Hilfe der Guajakblaureaktion der Peroxydasegehalt bezw. die Peroxydasewirkung auf etwa $10 \%$ genau bestimmen.

Mittels dieser Methode wurde die Peroxydase aus Cochlearia armoracia untersucht.

2. Die genannte Reaktion wird von der Acidität der Lösung innerhalb der Grenzen $+\mathrm{H}=10^{-2}$ bis $-\mathrm{OH}=10^{-2}$ sehr wenig beeinflußt; die Gifte Chloroform, Formaldehyd, Chininsulfat schädigen in verdünnter Lösung nicht.

3. Als Reinigungsmethode für die Präparate von Cochlearia-Peroxydase eignet sich Dialyse weit besser als fraktionierte Fällung durch Alkohol. Im besten, d. h. pro Gewichtseinheit wirksamsten Präparat, welches die Verfasser bis jetzt erhalten haben, wurden $10,4 \% \mathrm{~N}$ und 2,5\% Asche gefunden.

4. Die Wärmeempfindlichkeit der untersuchten Peroxydase wurde gemessen. Bei $100^{\circ}$ sinkt die Wirksamkeit schon in Bruchteilen einer Minute auf die Hälfte.

5. Quantitative Vergleiche zeigen, daß man die Peroxydasewirkung nicht einfach auf die Gegenwart dreiwertigen Eisens und nicht auf diejenige von Chinonen zurückführen kann; bekanntlich rufen diese Stoffe ebenfalls die Guajakblaureaktion hervor. Es wären zur quantitativen Erreichung der Peroxydasewirkung viel größere Eisen- bezw. Chinonmengen erforderlich, als sich tatsächlich vorfinden. Man ist vollständig berechtigt, die Peroxydase zu den Enzymen zu zählen, und wird sie mit Chodat und Bach als wesentlichen Bestandteil der Oxydasen betrachten. 
II.

6. Die Rhus-Laccase ist von der Medicago-Laccase durchaus verschieden, sodaß sich eine Änderung in der Nomenklatur empfiehlt.

7. Wässerige, 1\%/ige Lösungen von Präparaten der RhusLaccase, nach Bertrand dargestellt, reagieren neutral; die Konzentration der Wasserstoffionen liegt zwischen $10^{-6}$ und $10^{-8}$. Die Oxydationskatalyse durch Rhus-Laccase kann keineswegs auf die gleichzeitige Gegenwart von Mangan und Hydroxylionen zurückgeführt werden. Die in dieser Richtung geäußerten Vermutungen können als endgültig erledigt betrachtet werden, und Bertrands Angaben über diese Oxydase bestätigen sich durchaus.

\section{Erwiderung auf L. Borchardts II. Mitteilung: Über die diabetische Lävulosurie und den qualitativen Nachweis der Lävulose im Harn. \\ Von}

Dr. Wilhelm Voit.

(Spezialarzt für Magen-, Darm- und Zuckerkranke in Nürnverg.)

(Der Redaktion zugegangen am 3. Juli 1909.)

L. Borchardt hat in dieser Zeitschrift eine Probe angegeben zum Nachweis der Lävulose in diabetischen Harnen; ${ }^{1}$ ) die von ihm mit dieser Probe angestellten Untersuchungen ergaben negative Resultate. Ich habe die Probe auf Veranlassung des Herrn Professor Sandmeyer in dessen Sanatorium für Zuckerkranke in Berlin-Zehlendorf voriges Jahr nachgeprüft und mit einer großen Mehrzahl diabetischer Harne positive Resultate erhalten, ja sogar mit normalen Harnen mehrfach zweifellos positive Resultate gefunden. Auf meine diesbezügliche Arbeit ${ }^{2}$ ) geht Borchardt in einer II. Mitteilung über diabetische Lävulosurie ein und bestreitet die Richtigkeit meiner Resultate. ${ }^{3}$ ) Darauf möchte ich folgendes erwidern:

1) Diese Zeitschrift, Bd. LV, Heft 3.

2) Diese Zeitschrift, Bd. LVIII, Heft 2.

3) Diese Zeitschrift, Bd. LX, Heft 5. 